\title{
Metastatic pulmonary calcification as a rare complication of end-stage renal disease with coexistence of pulmonary metastases from renal cell carcinoma: case report and literature review
}

\author{
Marta Michali-Stolarska ${ }^{A, B, C, D, E, F}$, Anna Zacharzewska-Gondek ${ }^{A, B, C, D, E, F}$, Joanna Bladowska ${ }^{A, D, E}$, Maciej Guziński ${ }^{A, E}$, \\ Marek J. Sąsiadek ${ }^{\mathrm{A}, \mathrm{E}, \mathrm{G}}$
}

Department of General and Interventional Radiology and Neuroradiology, Wrocław Medical University, Wrocław, Poland

\section{Abstract}

\begin{abstract}
Purpose: We present a case of metastatic pulmonary calcification (MCP) in an asymptomatic patient with chronic kidney disease after renal transplantation and nephrectomy due to renal cancer. Chest computed tomography (CT) revealed bilateral, diffuse, centrilobular ground-glass opacities and heterogeneous, high-density areas distributed throughout the lungs, predominantly in the upper and middle lobes. Unusually, in our patient the metastatic calcification coexisted with pulmonary metastases from renal cell carcinoma associated with end-stage renal disease. To our knowledge, such coexistence has not been previously described.

Case report: CT, particularly high-resolution chest computed tomography (HRCT), plays an important role in detection and follow-up of MPC findings, which include ground-glass opacities and partially calcified nodules or consolidations, predominantly in the upper lung zones. Correct diagnosis is important because misdiagnosis may lead to improper or unnecessary treatment and/or procedures.
\end{abstract}

Conclusions: MPC is a rare condition that results from calcium deposition in the normal pulmonary parenchyma. MPC commonly occurs in patients with end-stage chronic kidney disease due to abnormalities in calcium and phosphate metabolism. It is worth pointing out that despite the fact that the condition is called metastatic, it is a relatively benign lung disease with a generally good long-term prognosis.

Key words: Renal cell carcinoma, diagnostic imaging, chronic kidney failure, kidney transplantation, lung diseases, multidetector computed tomography

\section{Introduction}

Metastatic pulmonary calcification (MPC) is a rare metabolic lung disease that is commonly associated with chronic renal failure; however, it is rarely diagnosed antemortem. Deposition of calcium salts in the interstitium and parenchyma of the lungs is a characteristic manifestation of MPC $[1,2]$.

MPC occurs usually due conditions causing hypercalcemia. It may have a benign or malignant etiology. Benign etiologies include end-stage chronic kidney disease, primary and secondary hyperparathyroidism, exces- sive exogenous administration of calcium and vitamin D, osteoporosis, sarcoidosis, milk-alkali syndrome, Paget's disease, renal [1] or liver transplantation [1,3], and cardiac surgery [4].

Among malignant etiologies, multiple myeloma is the most common cause of cancer-related hypercalcemia, which is seen in $20-30 \%$ of cases $[5,6]$. Rarely, MPC occurs in patients with leukemia $[7,8]$. Other malignant causes include parathyroid carcinoma [5,9], lymphoma, breast carcinoma, choriocarcinoma, malignant melanoma, synovial carcinoma, and hypopharyngeal squamous cell carcinoma $[1,5]$.

Correspondence address:

Marta Michali-Stolarska, Department of General Radiology, Interventional Radiology and Neuroradiology, Wrocław Medical University, 213 Borowska St.,

50-556 Wrocław, Poland, e-mail: marta.michali@gmail.com

Authors' contribution:

A Study design · B Data collection · C Statistical analysis · D Datainterpretation - E Manuscript preparation · $F$ Literature search · G Funds collection 

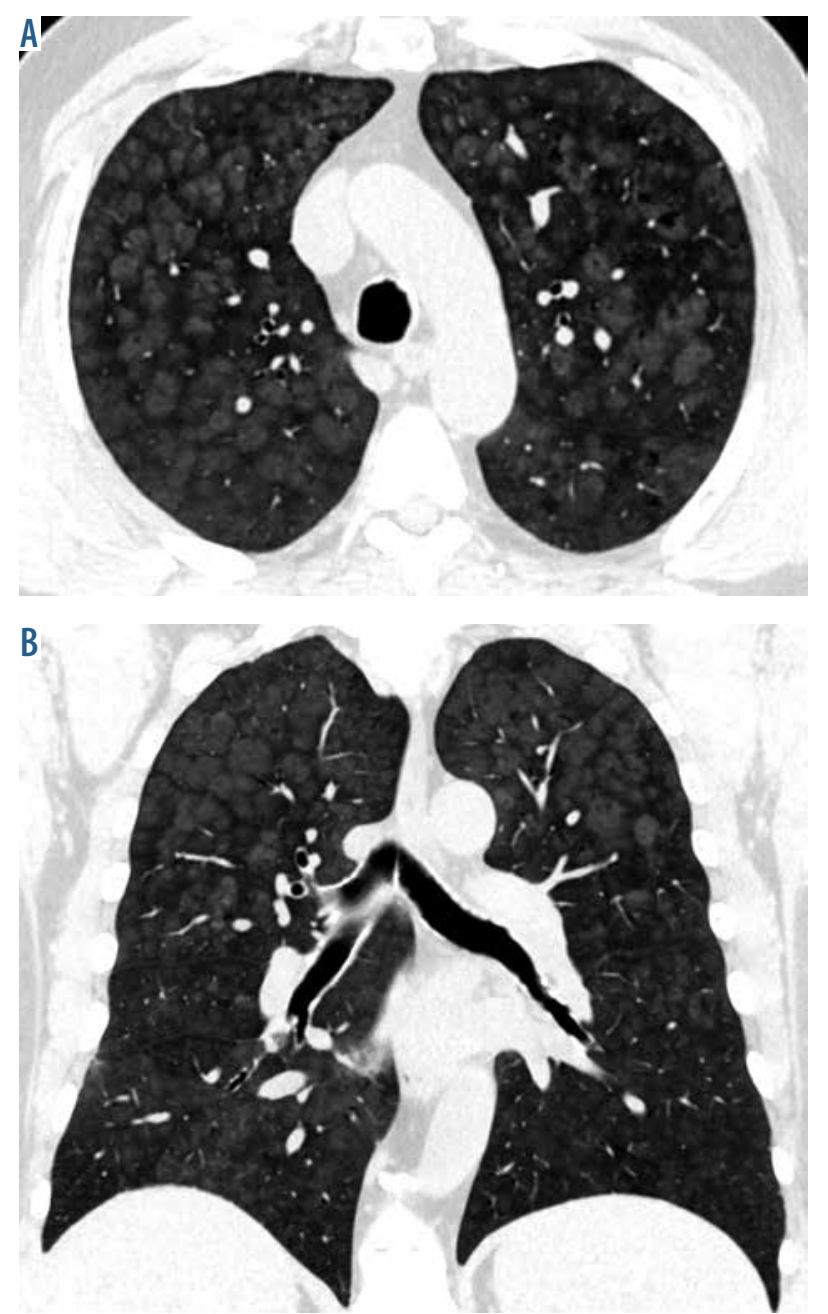

Figure 1. High-resolution chest computed tomography of the lungs. Metastatic pulmonary calcification: A) axial scan of the upper lungs shows bilateral, symmetrical, diffuse, indistinct, centrilobular ground-glass opacities with sparing of the periphery of the lobule and interlobular septa, B) coronal reformation shows predominantly upper and middle lung distribution of opacities

The majority of patients with MPC are asymptomatic, but several cases of respiratory failure have been reported $[5,7,9]$.

Patients with most severe manifestations have risk factors of MPC including intermittent imbalance of acidosis and alkalosis due to dialysis, and imbalance of calcium and phosphorus metabolism due to hyperparathyroidism [2]. MPC may occasionally appear in patients with normal renal function, no underlying pulmonary disease, and normal calcium and phosphate levels [1].

Chest radiographs are not very sensitive in the detection of MPC, and are usually normal or demonstrate patchy or confluent airspace opacities [10]. On high-resolution computed tomography (HRCT), three patterns may be present as follows: patchy or diffuse areas of groundglass opacity or consolidation, multiple diffuse calcified nodules, and confluent high-attenuation parenchymal consolidation. These are relatively stable pulmonary changes, as opposed to infectious processes, and their resistance to medical therapy, in a clinical setting of elevated serum calcium levels, is of diagnostic value [1]. The aim of this report is to describe the radiological, clinical, and laboratory features in an asymptomatic patient with accidental MPC findings coexisting with pulmonary metastases from renal cell carcinoma. Moreover, we discuss this case in the context of the current literature.

\section{Case report}

A 55-year-old man with long-lasting chronic kidney disease due to glomerulonephritis and a 4-year history of hemodialytic treatment underwent renal transplantation. He also underwent parathyroidectomy due to severe hyperparathyroid osteopathy with symptoms of sensorimotor polyneuropathy, but the procedure did not relieve the symptoms.

The patient did not complain of fever, dyspnea or cough, there were no signs of an infectious focus, and previous plain chest radiographs were normal.

One of the routine follow-up abdominal ultrasound examinations, which was performed 14 years after renal transplantation, aroused the suspicion of renal tumor in the left cirrhotic kidney, which was confirmed on abdominal CT. After nephrectomy, a histological diagnosis of renal cell carcinoma (clear cell carcinoma) was made. Follow-up abdominal CT revealed renal metastases in the right lower lung lobe and HRCT was planned subsequently. HRCT confirmed the presence of nodules, most likely metastatic, located mainly at the base of the lungs. Moreover, HRCT showed bilateral, diffuse, centrilobular ground-glass opacities and heterogeneous high-density areas distributed throughout the lungs, predominantly in the upper and middle lobes, with no apparent thickening of the interlobular septa, hilar, or mediastinal lymph node enlargement (Figure 1). HRCT also showed vessel calcifications in the wall of the chest (Figure 2B) and bronchial calcifications (Figure 2A). The combination of pulmonary, vascular, and bronchial calcifications suggested metastatic pulmonary calcification.

Partial resection of the upper right pulmonary lobe was performed, which was most affected by the lesions. Both renal metastases and MPC were histologically confirmed. Afterwards, chemotherapy was initiated, and after four cycles of pazopanib follow-up HRCT revealed partial regression of the metastatic nodules; however MPC was stable.

Retrospectively, on previous abdominal CT examinations discrete, diffuse, centrilobular ground-glass opacities were also visible bilaterally in the lower parts of the lungs and had slightly worsened within 2 years (Figure 3 ).

The laboratory results performed over the previous years showed slight hypercalcemia (serum calcium levels up to 10.8 [normal, $<10.6 \mathrm{mg} / \mathrm{dl}$ ]) and hyperparathyroidism (parathyroid hormone levels up to $151.9 \mathrm{pg} / \mathrm{ml}$ [normal, $<72$ pg/ml]). Phosphate levels were normal. Kidney function tests showed mildly elevated urea (up to 80 $\mathrm{mg} / \mathrm{dl}$ ) and creatinine (up to $1.84 \mathrm{mg} / \mathrm{dl}$ ) levels, and reduced glomerular filtration rate (down to $41 \mathrm{ml} / \mathrm{min} / 1.73 \mathrm{~m}^{3}$ ). 

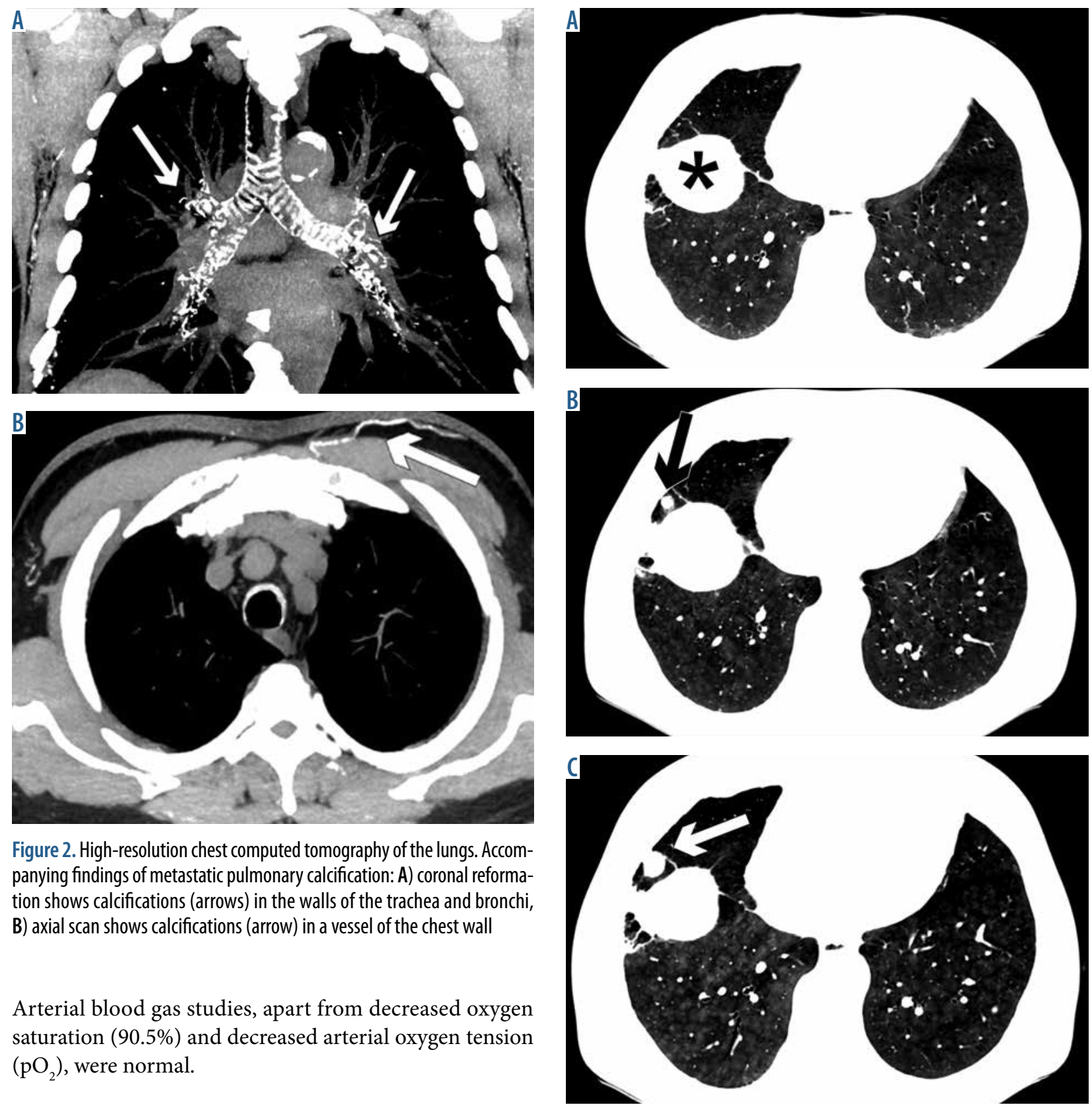

\section{Discussion}

MPC is an underdiagnosed metabolic lung disease that is commonly associated with chronic renal failure [2]. Histological changes of MPC are seen at autopsy in $60-75 \%$ of patients who were previously treated with hemodialysis, although the condition was rarely recognized antemortem, probably due to inadequate sensitivity of standard chest radiographs $[1,10]$. Pathologically, MPC is characterized by calcium salt deposition in basement membranes of the epithelium and endothelium of pulmonary alveoli. Soft tissue calcifications may also affect the stomach, heart, and kidneys, but the lung is the most frequently affected organ $[1,7,9]$.

Most patients with MPC are asymptomatic; however, this condition occasionally may cause severe dyspnea, chronic, non-productive cough, hypoxemia, hemoptysis, and rarely alveolar hemorrhage [2,11]. Several reports described acute respiratory failure that led to death $[5,7,9]$.

Figure 3. Follow-up computed tomography (CT) examinations. Slight progression of diffuse, centrilobular ground-glass opacities is visible bilaterally in the lower parts of the lungs due to metastatic pulmonary calcification, assessed retrospectively - A) baseline (T, B) follow-up (T after 1 year, C) follow-up high-resolution chest computed tomography after 2 years (arrows show progression of metastases of renal carcinoma, *diaphragm)

The severity of respiratory symptoms often does not correlate with the degree of macroscopic calcifications. Patients with extensive calcifications may have no respiratory symptoms, whereas those with minor changes or normal chest radiographs might sometimes have severe respiratory distress. Despite the fact that MPC generally develops over a long period of time, it can also progress rapidly within several weeks to months [2].

Metastatic, as opposed to dystrophic, pulmonary calcifications occur in normal pulmonary tissue without prior 
tissue damage, mainly due to alterations in calcium and phosphorus metabolism and an alkaline environment [1]. The primary pathogenic mechanism of MPC is still unexplained, but some factors, such as hypercalcemia (often due to hyperparathyroidism), hyperphosphatemia (as a result of hyperparathyroidism or reduced glomerular filtration rate), dialysis-associated intermittent acid-base imbalance, and local secretion of free hydrogen ions in the lungs, kidneys, and stomach have been suggested as the risk factors for MPC formation. Nevertheless, no correlation has been described with the levels of calcium or parathyroid hormone, history of parathyroidectomy, duration of hemodialysis, and type of dialysate [2,12].

Our patient had several factors that predisposed to MPC including hypercalcemia, hyperparathyroidism, chronic kidney failure, and history of hemodialysis.

Pulmonary CT, and particularly HRCT, has higher sensitivity for MPC detection than chest radiography, decreasing the need for open lung biopsy $[2,6]$. The pattern of involvement might show a predilection of metastatic calcifications for the upper lung lobes due to increased alkalinity at the apices, which leads to higher deposition of calcium salts in this location [2].

Several CT patterns of MPC have been documented to date, such as multiple, diffuse calcified nodules that are either distributed throughout the entire lung or show a predilection to the apices, diffuse or patchy areas of groundglass opacity or consolidation, and confluent, high-attenuation parenchymal consolidation with a predominantly lobar distribution, mimicking lobar pneumonia or edema. Bronchial wall thickening or inter-lobular septal thickening and tree-in-bud appearance are not observed in MPC [1].

The presence of centrilobular ground-glass nodular opacities, with multiple poorly defined and fluffy nodules measuring 3-10 $\mathrm{mm}$ in diameter, with or without foci of calcification, is the most common parenchymal finding on HRCT [10]. The peripheral regions of the alveoli tend to become alkalized in comparison to the central area, which may explain the characteristic distribution of ground-glass attenuation areas [1].

Vessel calcifications in the wall of the chest are frequently associated with MPC, and sometimes calcifications are also visible in the bronchial walls. A combination of pulmonary, vascular, and bronchial calcifications narrows down the differential diagnosis of pulmonary calcifications [10].

In our patient, HRCT showed typical MPC findings such as bilateral, diffuse, centrilobular ground-glass opacities and heterogeneous high-density areas distributed throughout the lungs, predominantly in the upper and middle lobes, vessel calcifications in the wall of the chest, and bronchial calcifications. The most unusual characteristic of our patient was the coexistence of metastatic calcification with pulmonary metastases from renal cell carcinoma of cirrhotic kidney. To our knowledge, this has not been previously described.

Radionuclide imaging with technetium-99m-methylene diphosphonate ( $\left.{ }^{99 \mathrm{~m}} \mathrm{Tc}-\mathrm{MDP}\right)$ is a sensitive and specific technique for an early detection of MPC [2]. Lung areas affected by MPC demonstrate increased and generally symmetrical uptake of the radioactive isotope [1]. Bronchoscopy performed in patients with MPC may reveal multiple nodular and linear lesions at the surface of the trachea and the main bronchi, with submucosal calcifications confirmed on biopsy $[13,14]$.

The majority of patients with asymptomatic, non-progressive MPC and renal failure do not require intervention. Although an optimal therapy of MPC has not been established, the mainstay of treatment is normalization of calcium and phosphate levels, primarily with phosphate binders. Furthermore, bisphosphonates are suggested to normalizethe calcium level and to stop progression of calcifications in hypercalcemic patients $[1,12]$. Parathyroidectomy is indicated in patients with life-threatening and uncontrollable pulmonary calcifications associated with hyperparathyroidism [9]. Renal transplantation may be considered for eligible patients. Several authors have noted reduction in calcifications, but there are also reports of dramatic progression of calcifications after transplantation [11], particularly after failed renal grafting [2,12].

The differential diagnosis of MPC includes all diseases that may lead to small, diffuse, calcified nodules, or small, diffuse, high-attenuation non-calcified nodules, and high-attenuation consolidations. Causes of small, diffuse, calcified nodules include infection (histoplasmosis and mycobacterial infections), pulmonary metastases (chondrosarcoma, osteosarcoma, mucin-producing adenocarcinomas, and papillary thyroid cancer), occupational and deposition diseases (coal workers' pneumoconiosis, silicosis, siderosis), chronic hemorrhagic conditions (hemosiderosis), and idiopathic disorders such as pulmonary alveolar microlithiasis [1]. If HRCT findings of MPC are diffuse, predominantly interstitial, and occasionally present as air-space opacities, they are often misdiagnosed as pneumonia or pulmonary edema. Lobar pneumonia, depending on its severity, may have a pattern of focal ground-glass opacity in a lobe or segment of the lung or dense opacification of the entire lobe. Similar bilateral patterns may appear in the course of pulmonary edema [3].

\section{Conclusions}

Metastatic pulmonary calcification (MPC) is often asymptomatic and underdiagnosed, which may lead to irreversible damage of the lungs and respiratory failure. Therefore, radiologists must be able to identify the imaging patterns of this condition. HRCT is the method of choice for detecting MPC, and may obviate the need for open lung biopsy. MPC should be considered when dialysis patients with chronic kidney failure develop unusual HRCT changes such as pulmonary infiltrates containing calcium depositions.

\section{Conflict of interest}

The authors report no conflict of interest. 


\section{References}

1. Belém LC, Zanetti G, Souza AS, et al. Metastatic pulmonary calcification: State-of-the-art review focused on imaging findings. Respir Med 2014; 108: 668-676.

2. Ueno K, Shimizu M, Uchiyama A, Hatasaki K. Fulminant respiratory failure due to progressive metastatic pulmonary calcification with no predisposing factors after successful renal transplantation: A case report. Pediatr Transplant 2016; 20: 1152-1156.

3. Sam JW. Case of the season. Metastatic pulmonary calcification following orthotopic liver transplant. Semin Roentgenol, 1999; 34: 253-255.

4. Sonavane S, Guleria S, Crowe DR, et al. Progressive lung calcification after orthotopic heart transplant. Pediatr Radiol 2015; 45: 124-128.

5. Cagirgan S, Soyer N, Vural F, et al. Metastatic pulmonary calcinosis and leukocytoclastic vasculitis in a patient with multiple myeloma. Turk J Haematol 2012; 29: 397-400.

6. Surani SR, Surani S, Khimani A, Varon J. Metastatic pulmonary calcification in multiple myeloma in a 45 -year-old man. Case Rep Pulmonol 2013; 2013: 341872.

7. Kumamoto H, Ichinohasama R, Sawai T, et al. Multiple organ failure associated with extensive metastatic calcification in a patient with an intermediate state of human T lymphotropic virus type I (HTLV-I) infection: Report of an autopsy case. Pathol Int 1998; 48: 313-318.
8. Cohen MC, Drut R. Metastatic pulmonary calcification with ossification in a child with acute lymphoblastic leukemia. Pediatr Pulmonol 1999; 27: 134-137.

9. Valdivielso P, López-Sánchez J, Garrido A, Sánchez-Carrillo JJ. Metastatic calcifications and severe hypercalcemia in a patient with parathyroid carcinoma. J Endocrinol Invest 2006; 29: 641-644.

10. Hartman TE, Muller NL, Primack SL, et al. Metastatic pulmonary calcification in patients with hypercalcemia: Findings on chest radiographs and CT scans. Am J Roentgenol 1994; 162: 799-802.

11. Georges S, Srour N. Metastatic pulmonary calcification in end-stage renal failure. Can Med Assoc J 2016; 188: E394.

12. Ando T, Mochizuki Y, Iwata T, et al. Aggressive pulmonary calcification developed after living donor kidney transplantation in a patient with primary hyperparathyroidism. Transplant Proc 2013; 45: 2825-2830.

13. Yasuo M, Tanabe T, Komatsu Y, et al. Progressive pulmonary calcification after successful renal transplantation. Intern Med 2008; 47: 161-164.

14. Ishii H, Kai N, Kashima K, et al. Bronchoscopic and electron microscopic findings in a dialysis patient with metastatic pulmonary calcification. Intern Med 2008; 47: 2099-2100. 